\title{
Three-dimensional Solid Element Based on the Finite Element Absolute Nodal Coordinate Formulation
}

\author{
Ma Chao*, Liu Hao, Zhao Yang \\ Department of Aerospace Engineering, Harbin Institute of Technology, Harbin, 150001, China \\ machaodn@163.com
}

Keywords: Flexible multibody system, Absolute nodal coordinate formulation, Solid element, Large deformation

\begin{abstract}
In multibody system dynamics, the absolute nodal coordinate formulation (ANCF) uses power functions as interpolating polynomials to describe displacement field. It can get accurate results for flexible bodies that undergo large-deformation and large-rotation. In this paper, an eight-node ANCF solid element has been provided and achieved. The continuity equations have been used to maintain the consistency for multiple elements, and the viscosity model has been proposed for the large deformations. The constraint equations and motion equations are developed based on the continuum mechanics theory for the solid element. Numerical results are presented to validate the feasibility of proposed element. It is shown that the proposed element can lead to a better convergence in comparison with the conventional finite elements in the dynamic analysis.
\end{abstract}

\section{Introduction}

As discussed in literatures, the conventional dynamic modeling methods based on small-deformation and small-rotation cannot get accurate results of large deformation flexible multibody problems [1]. The absolute nodal coordinate formulation (ANCF) was proposed by Shabana [2], and has been already used during the past decade to integrate finite elements into multibody system algorithms successfully. Olshevskiy [3] proposed a solid element based on the ANCF element firstly. Different from conventional finite elements, the ANCF solid element directly describes the section deformation using the node coordinates without bringing in additional coordinates, and the locking problems can also be avoided by using the incomplete interpolation function which used to describe the displacement field. Based on the above researches, the ANCF solid element considering the continuity condition and internal viscoelastic damping has been provided and achieved in this paper. Numerical examples are presented in order to demonstrate the feasibility of implementing element developed in this investigation.

\section{ANCF Solid Element}

Displacement Field. In the reference configuration, the global position vector of an arbitrary point on the element can obtain by using the global shape function and element absolute nodal coordinates. The relationship can be written as follows [4]:

$$
\boldsymbol{r}=\boldsymbol{S}(x, y, z) \boldsymbol{e}(t)
$$

where $\boldsymbol{S}$ is the shape function matrix; $x, y$ and $z$ are the node position coordinates; $\boldsymbol{e}$ is the vector of nodal coordinates.

The dimensions of the solid element are $a \times b \times c$, and the nodal coordinates $e$ at the node $k$ of the finite element $j$ can be defined as:

$$
\boldsymbol{e}^{j k}=\left[\boldsymbol{r}^{j k^{\mathrm{T}}} \boldsymbol{r}_{x}^{j k^{\mathrm{T}}} \boldsymbol{r}_{y}^{j k^{\mathrm{T}}} \boldsymbol{r}_{z}^{j k^{\mathrm{T}}}\right]^{\mathrm{T}} \quad k=1, \cdots, 8
$$

where $\boldsymbol{r}^{j k}$ is the absolute position vector at the node $k$ of the finite element $j$, and $\boldsymbol{r}_{x}^{j k}, \boldsymbol{r}_{y}^{j k}$ and $\boldsymbol{r}_{z}^{j k}$ are the position gradients vectors. The ANCF solid element is shown in Fig. 1. 


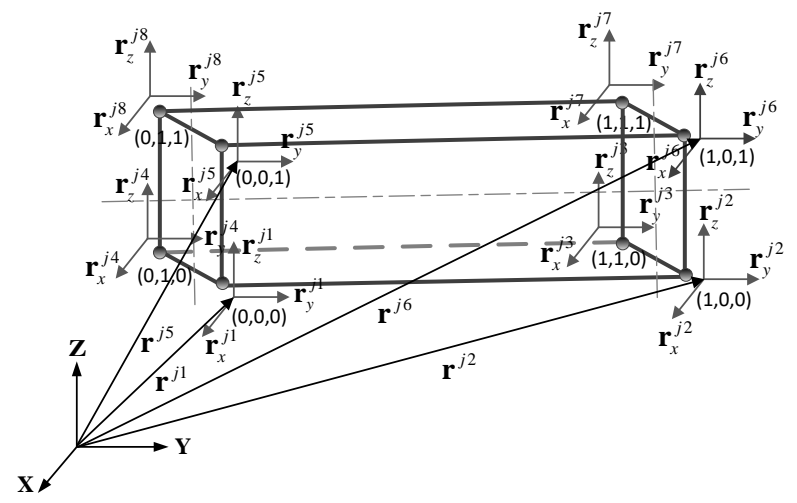

Fig. 1 Eight-node solid element

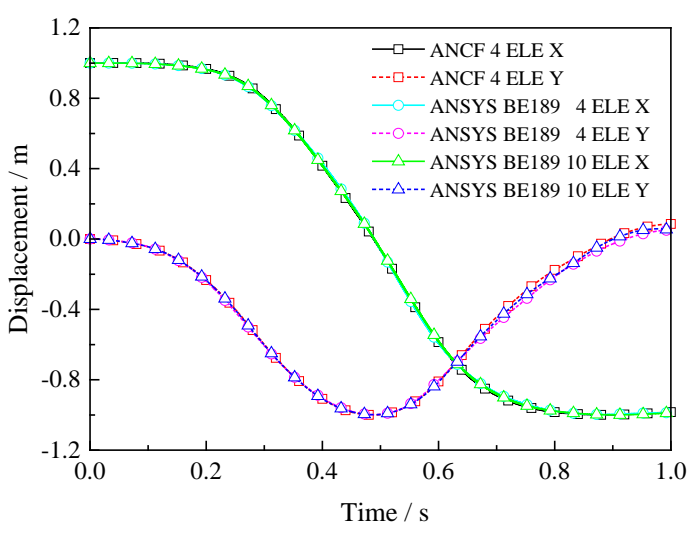

Fig. 2 Displacement of the simple pendulum at the end point

The displacement field of solid element can be defined as:

$$
\begin{aligned}
\phi(x, y, z)= & \alpha_{1}+\alpha_{2} x+\alpha_{3} y+\alpha_{4} z+\alpha_{5} x^{2}+\alpha_{6} y^{2}+\alpha_{7} z^{2}+\alpha_{8} x y+\alpha_{9} y z+\alpha_{10} x z+\alpha_{11} x^{3}+ \\
& \alpha_{12} y^{3}+\alpha_{13} z^{3}+\alpha_{14} x^{2} y+\alpha_{15} x^{2} z+\alpha_{16} y^{2} z+\alpha_{17} x y^{2}+\alpha_{18} x z^{2}+\alpha_{19} y z^{2}+ \\
& \alpha_{20} x y z+\alpha_{24} x^{3} y+\alpha_{25} x^{3} z+\alpha_{26} x y^{3}+\alpha_{27} y^{3} z+\alpha_{28} x z^{3}+\alpha_{29} y z^{3}+\alpha_{30} x^{2} y z+ \\
& \alpha_{31} x y^{2} z+\alpha_{32} x y z^{2}+\alpha_{36} x^{3} y z+\alpha_{37} x y^{3} z+\alpha_{38} x y z^{3}
\end{aligned}
$$

where $\alpha_{k}$ are the polynomial coefficients. The components of the shape function matrix of the ANCF solid element can be derived as follows:

$$
\left\{\begin{array}{rl}
S^{k, 1}= & (-1)^{1+\xi_{k}+\eta_{k}+\zeta_{k}}\left(\xi+\xi_{k}-1\right)\left(\eta+\eta_{k}-1\right)\left(\zeta+\zeta_{k}-1\right) \cdot \\
& \left(1+\left(\xi-\xi_{k}\right)(1-2 \xi)+\left(\eta-\eta_{k}\right)(1-2 \eta)+\left(\zeta-\zeta_{k}\right)(1-2 \zeta)\right) \\
S^{k, 2}= & (-1)^{\eta_{k}+\zeta_{k}} a \xi^{\xi_{k}+1}(\xi-1)^{2-\xi_{k}} \eta^{\eta_{k}}(\eta-1)^{1-\eta_{k}} \zeta^{\zeta_{k}}(\zeta-1)^{1-\zeta_{k}} \\
S^{k, 3}= & (-1)^{\xi_{k}+\zeta_{k}} b \xi^{\xi_{k}}(\xi-1)^{1-\xi_{k}} \eta^{\eta_{k}+1}(\eta-1)^{2-\eta_{k}} \zeta^{\zeta_{k}}(\zeta-1)^{1-\zeta_{k}} \\
S^{k, 4}= & (-1)^{\xi_{k}+\eta_{k}} c \xi^{\xi_{k}}(\xi-1)^{1-\xi_{k}} \eta^{\eta_{k}}(\eta-1)^{1-\eta_{k}} \zeta^{\zeta_{k}+1}(\zeta-1)^{2-\zeta_{k}}
\end{array} \quad k=1, \cdots, 8\right.
$$

where $a, b$ and $c$ are the dimensions of the element along the $x, y$ and $z, \xi_{k}, \eta_{k}$ and $\zeta_{k}$ are the dimensionless coefficients. The position vector of an arbitrary material point can be written as:

$$
\boldsymbol{r}^{j}=\sum_{k=1}^{8}\left[\begin{array}{llll}
S^{k, 1} \boldsymbol{I}_{3 \times 3} & S^{k, 2} \boldsymbol{I}_{3 \times 3} & S^{k, 3} \boldsymbol{I}_{3 \times 3} & S^{k, 4} \boldsymbol{I}_{3 \times 3}
\end{array}\right] \boldsymbol{e}^{j k}
$$

where $\boldsymbol{S}^{j}$ and $\boldsymbol{e}^{j}$ are , respectively, the element global shape function matrix and the vector of nodal coordinates.

Continuity Condition. It is known that the ANCF finite element can automatically ensure $C^{1}$ continuity in the interface between displacement fields. The nodes on the adjacent surface between element $p$ and $q$ can meet the $C^{0}, C_{y}^{1}$ and $C_{z}^{1}$ continuous. The $C_{x}^{1}$ continuous needs to satisfy in order to guarantee the multi-element continuously:

$$
\boldsymbol{S}_{x}(\xi=1) \boldsymbol{e}^{p}=\boldsymbol{S}_{x}(\xi=0) \boldsymbol{e}^{q}
$$

where $S_{x}$ is the derivative of element shape function $\boldsymbol{S}$ in $x$ direction, and $\boldsymbol{e}$ is the vector of nodal coordinates. The Equation 6 can be written as

$$
\left\{\begin{array}{l}
\boldsymbol{r}_{x y}^{p m}=\boldsymbol{r}_{x y}^{q n} \\
\boldsymbol{r}_{x z}^{p m}=\boldsymbol{r}_{x z}^{q n}
\end{array} \quad m=2,3,6,7 \quad n=1,4,5,8\right.
$$

where $\boldsymbol{r}_{x y}^{p m}$ is the partial derivatives of the node $m$ from the element $p$ along $x$ and $y$ directions, $m$ and $n$ are, respectively, the sharing node on the adjacent surface between element $p$ and $q$.

Constitutive Equations. Describe the strain using the Green-Lagrange strain tensor in reference configuration as follows:

$$
\boldsymbol{\varepsilon}=\frac{1}{2}\left(\boldsymbol{J}^{\mathrm{T}} \boldsymbol{J}-\boldsymbol{I}\right)
$$


where $\varepsilon$ is Green-Lagrange strain tensor, and $\boldsymbol{J}$ is deformation gradient. In reference configuration, describe the elastic stress using the second Piora-Kirchhoof stress tensor as follows:

$$
\boldsymbol{\sigma}_{P 2}=J \boldsymbol{J}^{-1} \boldsymbol{\sigma} \boldsymbol{J}^{-1^{\mathrm{T}}}
$$

where $\sigma_{P 2}$ is the second Piora-Kirchhoof stress tensor, and $\sigma$ is Cauchy stress tensor in current configuration. The relationship of strain and stress are

$$
\boldsymbol{\sigma}_{P 2, v}=\boldsymbol{E} \boldsymbol{\varepsilon}_{v}
$$

where $\boldsymbol{E}$ is material coefficients matrix.

Define the relationship between damping stress and the rate of change of the strain tensor as follows:

$$
\sigma_{d, v}=D \dot{\boldsymbol{\varepsilon}}_{v}
$$

where $\sigma_{d}$ is damping stress, and $\dot{\boldsymbol{\varepsilon}}$ is the rate of change of the Green-Lagrange strain tensor. $\boldsymbol{D}$ is material damping coefficients matrix.

Dynamics Equations. According to the principle of virtual work, the elastic force of element in reference configuration can be obtained by Green-Lagrange strain tensor and the second Piola-Kirchhoff stress tensor as follows:

$$
\delta W_{k e}^{j}=-\int_{V} \boldsymbol{\sigma}_{P 2}^{j}: \delta \varepsilon^{j} \mathrm{~d} V=-\boldsymbol{Q}_{k e}^{j} \cdot \delta \boldsymbol{e}^{j}
$$

where $\delta W_{k e}^{j}$ is the virtual work of elastic force, and $\boldsymbol{Q}_{k e}^{j}$ is generalized elastic force.

For damping force, it can be obtained from the rate of change of the Green-Lagrange strain tensor and damping stress as follows:

$$
\delta W_{k d}^{j}=-\left(\int_{V} \boldsymbol{\sigma}_{d}^{j}: \delta \dot{\boldsymbol{\varepsilon}}^{j} \mathrm{~d} V\right) \mathrm{d} t=-\boldsymbol{Q}_{k d}^{j} \cdot \delta \boldsymbol{e}^{j}
$$

where $\delta W_{k d}^{j}$ is the virtual work of damping force, and $\boldsymbol{Q}_{k d}^{j}$ is generalized damping force.

The virtual work of the inertia forces is defined as

$$
\delta W_{I}^{j}=\int_{v} \rho_{0} \boldsymbol{a}^{j^{\mathrm{T}}} \cdot \delta \boldsymbol{r}^{j} \mathrm{~d} v=\boldsymbol{Q}_{I}^{j} \cdot \delta \boldsymbol{e}^{j}=\boldsymbol{M}^{j} \ddot{\boldsymbol{e}}^{j} \cdot \delta \boldsymbol{e}^{j}
$$

where $\delta W_{I}^{j}$ is the virtual work of inertial force, and $\boldsymbol{a}^{j}$ is acceleration vector of element, $\boldsymbol{Q}_{I}^{j}$ is generalized inertial force, $\boldsymbol{M}^{j}$ is a constant symmetric mass matrix, $\boldsymbol{M}^{j}=\int_{V} \rho \boldsymbol{S}^{j^{\mathrm{T}}} \boldsymbol{S}^{j} \mathrm{~d} V$.

For the external force, it can be obtained by principle of virtual work as follows:

$$
\delta W_{e}^{j}=\int_{V} f^{j^{\mathrm{T}}} \cdot \delta \boldsymbol{r}^{j} \mathrm{~d} V=\boldsymbol{Q}_{e}^{j} \cdot \delta \boldsymbol{e}^{j}
$$

where $\delta \mathrm{W}_{e}^{j}$ is the virtual work of external force, $\boldsymbol{f}^{j}$ is external force, and $\boldsymbol{Q}_{e}^{j}$ is generalized external force.

The dynamics equation of multibody system is written as:

$$
\delta \boldsymbol{q}^{\mathrm{T}}\left(\boldsymbol{M} \ddot{\boldsymbol{q}}+\boldsymbol{C}_{\boldsymbol{q}}^{\mathrm{T}} \boldsymbol{\lambda}-\boldsymbol{Q}\right)=0
$$

where $\boldsymbol{M}$ is mass matrix of the system, $\boldsymbol{q}$ is generalized coordinate of the system, $\boldsymbol{C}_{\boldsymbol{q}}$ is the matrix of constraint equation, $\lambda$ is the vector of Lagrange multipliers, $\boldsymbol{Q}$ is the generalized force of the system.

\section{Numerical Examples}

Simple Pendulum. The pendulum is constrained by spherical joints at the end of one side, in which the translation displacements of the node are fixed and the rotations are free. The dimensions of beam are $1.0 \mathrm{~m} \times 0.02 \mathrm{~m} \times 0.02 \mathrm{~m}$. The density of the material $\rho=7200 \mathrm{~kg} / \mathrm{m}^{3}$. The gravity acceleration $g=9.8 \mathrm{~m} / \mathrm{s}^{2}$. The modulus of elasticity $E=2.0 \mathrm{e} 8 \mathrm{~Pa}$. The Poisson's ratio $v=0.3$. The beam is in a static at the initial and rotates freely under the gravity when the simulation begins. The displacements at the end point of simple pendulum are shown in Fig. 2.

From the Fig. 2, it can be found that, the displacement curves obtained from ANCF solid elements are almost identical with curves obtained from BEAM189 elements. The results illustrate that the solid element show a good adaptability to describe the deformation, and the capacity of the ANCF solid element in dealing with flexible deformations is equal to the conventional element. 
Double Pendulum. The pendulum is constrained by spherical joints at the end of one side. The two beams of the double pendulum have the same parameters. The dimensions of each beam are $1.0 \mathrm{~m} \times 0.02 \mathrm{~m} \times 0.02 \mathrm{~m}$. The density of the material $\rho=7200 \mathrm{~kg} / \mathrm{m}^{3}$. The gravity acceleration $g=9.8 \mathrm{~m} / \mathrm{s}^{2}$. The modulus of elasticity $E=2.0 \mathrm{e} 7 \mathrm{~Pa}$. The Poisson's ratio $v=0.3$. The displacements at the end points from the upper and lower beams using the ANCF solid elements and ANSYS BEAM189 elements are compared in Fig. 3 and Fig. 4.

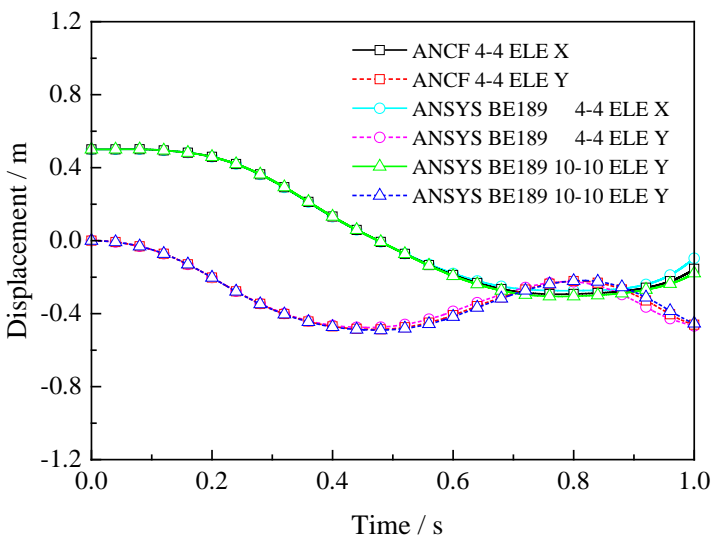

Fig. 3 Displacement of the upper pendulum at the end points

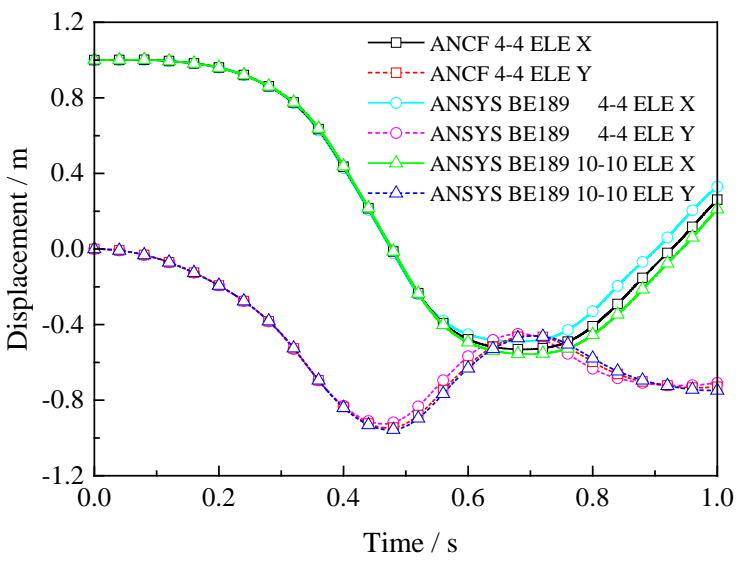

Fig. 4 Displacement of the lower pendulum at the end points

The difference of the displacement curves shows that, the displacement curves obtained from ANCF solid elements and BEAM189 elements are almost identical at the initial time. As the simulation goes on, the curves of 10 BEAM189 elements approach to the curves of ANCF solid elements gradually. The difference of the displacement curves shows that, the advantages of ANCF solid finite element are fully embodied in the large deformation problems.

\section{Summary}

In this paper, an eight-node three-dimensional ANCF solid element has been developed in this investigation. In this model, the continuity condition has been used to apply the algebraic constraint equations to maintain the continuity at the boundary. Using the relationship between the deformation gradients and the components of the Green-Lagrange strain tensor, the viscoelastic model is developed based on the Kelvin-Voigt model for the ANCF solid element. The feasibility of implementing the solid element is demonstrated using numerical examples. The results obtained from the ANCF solid elements are compared with the conventional finite elements. The comparisons show a good agreement in the dynamic analysis. Furthermore, the ANCF solid element can get higher accuracy than conventional finite element for large deformation analysis.

\section{References}

[1] A. A. Shabana, Flexible multibody dynamics: Review of past and recent developments[J]. Multibody System Dynamics, 1997, 1(2): 189-222.

[2] A. A. Shabana, Definition of the slopes and the finite element absolute nodal coordinate formulation[J]. Multibody System Dynamics, 1997, 1(3): 339-348.

[3] A. Olshevskiy, O. Dmitrochenko, C. W. Kim, Three-dimensional solid brick element using slopes in the absolute nodal coordinate formulation[J]. Journal of Computational and Nonlinear Dynamics, 2014, 9(2): 021001-1-021001-10.

[4] A. A. Shabana, Dynamics of multibody systems[M]. 3rd ed. Cambridge/New York: Cambridge University Press, 2005: 267-304. 